\title{
Planning rigid body motions using Elastic curves
}

\author{
James Biggs and William Holderbaum
}

\begin{abstract}
This paper tackles the problem of computing smooth, optimal trajectories on the Euclidean group of motions $S E(3)$. The problem is formulated as an optimal control problem where the cost function to be minimized is equal to the integral of the classical curvature squared. This problem is analogous to the elastic problem from differential geometry and thus the resulting rigid body motions will trace elastic curves. An application of the Maximum Principle to this optimal control problem shifts the emphasis to the language of symplectic geometry and to the associated Hamiltonian formalism. This results in a system of first order differential equations that yield coordinate free necessary conditions for optimality for these curves. From these necessary conditions we identify an integrable case and these particular set of curves are solved analytically. These analytic solutions provide interpolating curves between an initial given position and orientation and a desired position and orientation that would be useful in motion planning for systems such as robotic manipulators and autonomous oriented vehicles.
\end{abstract}

\section{INTRODUCTION}

There are a number of applications in which the problem of generating smooth $3-D$ trajectories for a rigid body is encountered. For example in the path planning problem for autonomous oriented vehicles such as Underwater Vehicles [1], Unmanned Air Vehicles [2], for simulating objects in computer graphics [3] and trajectory generation in robotics and kinematics [4]. In each of these problems it is desirable to plan a smooth motion between an initial position and orientation and a desired final position and orientation in $\mathbb{R}^{3}$. In addition to planning smooth motions it is desirable that the trajectories be invariant with respect to the choice of coordinate system used to describe the motion. In particular screw motion forms the basis for motion planning schemes in [5] and [6]. Although screw displacement is smooth and invariant with respect to rigid body transformations, it does not optimize a meaningful cost function. In this paper we aim to plan smooth trajectories that can be expressed independently of the coordinate frame and are also optimized with respect to some meaningful cost function.

Various literature has tackled the optimal trajectory planning problem using a variational approach and optimizing the curve with respect to some practically motivated cost function. For example in [7], [8] the authors derive coordinate free necessary conditions for generalized cubic splines. In [9], [10] for systems defined on $S E(3)$, a coordinate free

This work was undertaken at the University of Reading, James Biggs is currently with the Mechanical Engineering Department, University of Strathclyde, Glasgow. james.biggs@strath.ac.uk

W. Holderbaum is with School of Systems Engineering, University of Reading, Reading, The United Kingdom w. holderbaumerdg.ac.uk formulation of the variational approach was used to generate shortest paths, minimum acceleration and jerk trajectories.

In this paper we propose a method that generates smooth $3-D$ trajectories that minimize the integral of the square of the curvature of the curve. This problem is analogous to the elastic problem from differential geometry described in [11]. Therefore, rigid body systems which trace these smooth, optimal curves will trace elastic curves in Euclidean space. Such a cost function is practically motivated as it minimizes the amount of steering required to track such a curve and therefore avoids using high accelerations and forces. In addition this paper uses the Maximum Principle of Optimal control rather than a direct variational approach [7], [8] to solve these problems. This method results in a Hamiltonian system which lends itself to conceptually clear global investigations. The advantage of the Hamiltonian setting is that we can directly apply the theory of integrable Hamiltonian systems [12]. If the conditions of this theorem are satisfied then analytic solutions exist and it is therefore of significance to try and solve for them. In addition the presence of several conserved quantities inherent in these Hamiltonian systems aids in the explicit computation of the rigid body motions.

The motion planning problem for rigid body systems is formulated as an optimal control problem on the Lie group $S E(3)$, where the cost function to be minimized is the integral of the curvature squared (analogous to the elastic energy in [11]). The coordinate free Maximum Principle [12], [13] is then applied to solve this problem. The emphasis of this paper is placed on an integrable case where the necessary conditions for optimality can be expressed analytically. In addition the corresponding optimal motions are expressed in a coordinate free manner, that is they are described completely in terms of the geometrically invariant natural curvatures. These optimal motions are shown to trace helical paths which could be useful in motion interpolation schemes. This problem formulation is both practical for the path planning application considered and illuminates how the general theory of optimal control, framed curves and left-invariant Hamiltonian systems applies to this particular setting.

\section{NATURAL FRAMES}

In order to compute smooth trajectories in $S E(3)$ we assign a geometric frame to the rigid body system, that is, a moving frame along a space curve. Each point on the moving rigid body traces out a curve in space and hence has a Serret-Frenet frame associated with it. An orthonormal frame attached to the rigid body stays aligned with the SerretFrenet frame and the parameters of motion are given by 
the curvature and torsion of the space curve along which the object moves. The Serret-Frenet frame has been used previously to study rigid body motions for robotic systems in [14] and [15]. It is essential to note that framed curves will in general not coincide with the rigid body fixed frame. However, in the particular case that the rigid body motion is constrained to travel in the direction of the unit tangent vector to the curve the two frames can be related [12]. Moreover, the rigid body is reduced to a particle and the Serret-Frenet frame describes the kinematics of this particle as it moves along a continuous, differentiable curve.

For existence and uniqueness of the Serret-Frenet frame the curve must be of class $C^{n}$ where $n \geq 3$ and the nondegeneracy condition of nonvanishing classical curvature must hold. At zero curvature the normal vector is not well-defined (it is arbitrary), hence it is not possible to associate a unique Serret-Frenet frame at such a point, nor uniquely continue it beyond this point. Such conditions have caused problems in formulating control laws in [2]. This problem was overcome in [2] by using an alternative frame known as the Natural frame [16]. The natural frame does not require the nondegeneracy condition and only that the curve be of class $C^{n}$ with $n \geq 2$.

The Natural Frame [16], whose parameters of motion are given by the natural curvatures of the space curve along which the object moves, has previously been suggested in the robotics literature [18] where these motions are referred to as 'rotation minimizing'. The Natural frame is useful in these applications as it does not twist about the curve as much as the Serret-Frenet frame. Additionally, rotation minimizing frames such as Fermi-Walker frames have been used extensively in the physics literature to model relativistic kinematics, see for example [19].

Explicitly, the Natural frame is defined by an orthonormal frame about the curve $\gamma(t)$ described by the following differential equations:

$$
\begin{aligned}
& \dot{\gamma}(t)=x \\
& \dot{x}=k_{1} y+k_{2} z \\
& \dot{y}=-k_{1} x \\
& \dot{z}=-k_{2} x
\end{aligned}
$$

where $\gamma(t) \in \mathbb{R}^{3}$, the orthonormal frame $R(t)=(x|y| z) \in$ $S O(3)$ and $k_{1}, k_{2}$ are the natural curvatures which are related to the classical curvature $\kappa$ of the Serret-Frenet frame by $\kappa^{2}=k_{1}^{2}+k_{2}^{2}$ [16]. In this paper we wish to use this Natural frame as well as the Maximum Principle of optimal control for systems defined on a Lie group [12] to plan rigid body motions. In order to formulate our problem statement in the context of an optimal control problem on the Lie group $S E(3)$, it is necessary to lift the Natural frame (1) to a differential system defined on $S E(3)$. This lift is similar to that for the Serret-Frenet frame explained in [12]. We define an element $g(t) \in S E(3)$ by:

$$
g(t)=\left(\begin{array}{cc}
1 & 0 \\
\gamma(t) & R(t)
\end{array}\right)
$$

and therefore is associated with (1) via the relations

$$
\begin{gathered}
{\left[\begin{array}{cc}
1 & \gamma(t)
\end{array}\right]^{T}=g(t) \vec{e}_{1}, \quad\left[\begin{array}{ll}
0 & x
\end{array}\right]^{T}=g(t) \vec{e}_{2}} \\
{\left[\begin{array}{ll}
0 & y
\end{array}\right]^{T}=g(t) \vec{e}_{3}, \quad\left[\begin{array}{ll}
0 & z
\end{array}\right]^{T}=g(t) \vec{e}_{4}}
\end{gathered}
$$

where $\vec{e}_{1}, \vec{e}_{2}, \vec{e}_{3}, \vec{e}_{4}$ is the standard orthonormal frame in $\mathbb{R}^{4}$. The Natural Frame (1) can then be expressed as a differential system on $S E(3)$ :

Proposition 1: The left-invariant differential equation:

$$
\frac{d g(t)}{d t}=g(t)\left(\begin{array}{cccc}
0 & 0 & 0 & 0 \\
1 & 0 & -k_{1} & -k_{2} \\
0 & k_{1} & 0 & 0 \\
0 & k_{2} & 0 & 0
\end{array}\right)
$$

where $g(t) \in S E(3)$ is equivalent to the Natural frame (1). Proof.

it follows from differentiating (3) w.r.t $t$ that

$$
\begin{aligned}
& {\left[\begin{array}{ll}
0 & \dot{\gamma}(t)
\end{array}\right]^{T}=\frac{d g(t)}{d t} \vec{e}_{1}=g(t) \vec{e}_{2}=\left[\begin{array}{ll}
0 & x
\end{array}\right]^{T}} \\
& {\left[\begin{array}{cc}
0 & \dot{x}
\end{array}\right]^{T}=\frac{d g(t)}{d t} \vec{e}_{2}=g(t)\left(k_{1} \vec{e}_{3}+k_{2} \vec{e}_{4}\right)=k_{1}\left[\begin{array}{ll}
0 & y
\end{array}\right]^{T}+k_{2}\left[\begin{array}{ll}
0 & z
\end{array}\right]^{T}} \\
& {\left[\begin{array}{ll}
0 & \dot{y}
\end{array}\right]^{T}=\frac{d g(t)}{d t} \vec{e}_{3}=g(t)\left(-k_{1} \vec{e}_{2}\right)=-k_{1}\left[\begin{array}{ll}
0 & x
\end{array}\right]^{T}} \\
& {\left[\begin{array}{ll}
0 & \dot{z}
\end{array}\right]^{T}=\frac{d g(t)}{d t} \vec{e}_{4}=g(t)\left(-k_{2} \vec{e}_{2}\right)=-k_{2}\left[\begin{array}{ll}
0 & x
\end{array}\right]^{T}}
\end{aligned}
$$

then equating the L.H.S to the R.H.S yields (1).

The system (4) can be expressed conveniently in coordinate form by defining the following basis for $\mathfrak{s e}(3)$

$$
\begin{aligned}
A_{1} & =\left(\begin{array}{cccc}
0 & 0 & 0 & 0 \\
0 & 0 & -1 & 0 \\
0 & 1 & 0 & 0 \\
0 & 0 & 0 & 0
\end{array}\right), A_{2}=\left(\begin{array}{cccc}
0 & 0 & 0 & 0 \\
0 & 0 & 0 & -1 \\
0 & 0 & 0 & 0 \\
0 & 1 & 0 & 0
\end{array}\right) \\
A_{3} & =\left(\begin{array}{cccc}
0 & 0 & 0 & 0 \\
0 & 0 & 0 & 0 \\
0 & 0 & 0 & -1 \\
0 & 0 & 1 & 0
\end{array}\right), B_{1}=\left(\begin{array}{cccc}
0 & 0 & 0 & 0 \\
0 & 0 & 0 & 0 \\
0 & 0 & 0 & 0 \\
1 & 0 & 0 & 0
\end{array}\right) \\
B_{2} & =\left(\begin{array}{cccc}
0 & 0 & 0 & 0 \\
0 & 0 & 0 & 0 \\
1 & 0 & 0 & 0 \\
0 & 0 & 0 & 0
\end{array}\right), B_{3}=\left(\begin{array}{cccc}
0 & 0 & 0 & 0 \\
1 & 0 & 0 & 0 \\
0 & 0 & 0 & 0 \\
0 & 0 & 0 & 0
\end{array}\right)
\end{aligned}
$$

and it follows that (4) can be expressed as:

$$
\frac{d g(t)}{d t}=g(t)\left(B_{3}+k_{1} A_{1}+k_{2} A_{2}\right)
$$

As explained in the introduction we will compute smooth trajectories, that minimize the integral of the classical curvature squared, which is equivalent to minimizing the elastic energy of the curve [11]. For the Natural frame (7) this is equivalent to minimizing the function:

$$
J=\frac{1}{2} \int \kappa^{2} d t=\frac{1}{2} \int\left(k_{1}^{2}+k_{2}^{2}\right) d t
$$


These natural curvatures are analogous to the gyroscopic steering controls for an Unmanned Air Vehicle, see [2]. This problem is formally stated as an optimal control problem:

Problem Statement 1: Compute the motions $g(t) \in S E(3)$ of the left-invariant differential system:

$$
\frac{d g(t)}{d t}=g(t)\left(B_{3}+k_{1} A_{1}+k_{2} A_{2}\right)
$$

that minimizes the expression:

$$
J=\frac{1}{2} \int\left(k_{1}^{2}+k_{2}^{2}\right) d t
$$

subject to the given boundary conditions $g(0)=g_{0}$ and $g(T)=g_{T}$, where $k_{1}, k_{2}$ are the natural (steering) curvatures. The Problem Statement 1 is geometrically analogous to the elastic problem in [11]. It follows that the rigid body motions that correspond to Problem Statement 1 will trace elastic curves in Euclidean space. In the following section we apply the Maximum Principle to Problem Statement 1.

\section{Hamiltonian LifT ON SE(3)}

The application of the Maximum Principle [13], [12] to Problem Statement 1 shifts the emphasis to the language of symplectic geometry and to the associated Hamiltonian formalism. The Maximum Principle states that the optimal paths are the projections of the extremal curves onto the base manifold, where the extremal curves are solutions of certain Hamiltonian systems on the cotangent bundle. In Problem Statement 1 the manifold in question is $S E(3)$ and the cotangent bundle is $T^{*} S E(3)$. We begin by defining the appropriate pseudo-Hamiltonian on $T^{*} S E(3)$ for Problem Statement 1 (see [24], [12] for details):

$$
\begin{gathered}
H(p, u, g)=p\left(g(t) B_{1}\right)+k_{1} p\left(g(t) A_{1}\right)+k_{2} p\left(g(t) A_{2}\right) \\
-\rho_{0} \frac{1}{2}\left(k_{1}^{2}+k_{2}^{2}\right)
\end{gathered}
$$

where $p(\cdot): T S E(3) \mapsto \mathbb{R}$ and $\rho_{0}=1$ for regular extremals and $\rho_{0}=0$ for abnormal extremals. The abnormal extremals arise as solutions of the optimal control problem defined by the constraints alone and are the subject of extreme interest, see [20], [21]. These situations are not of principle concern in this paper and we consider only the regular extremals $\left(\rho_{0}=1\right)$. As the configuration of the rigid body is the Lie group $S E(3)$, the cotangent bundle $T^{*} S E(3)$ can be realized as the direct product $S E(3) \times \mathfrak{s e}^{*}(3)$ where $\mathfrak{s e}^{*}(3)$ is the dual of the Lie algebra $\mathfrak{s e}(3)$ of $S E(3)$, see [12]. Therefore, the original Hamiltonian defined on $T^{*} S E(3)$ can be expressed as a reduced Hamiltonian on the dual of the Lie algebra $\mathfrak{s e}^{*}(3)$. We define the linear functions $M_{i}=\hat{p}\left(A_{i}\right), p_{i}=\hat{p}\left(B_{i}\right)$ for $i=1,2,3$ where $\hat{p}(\cdot): \mathfrak{s e}(3) \mapsto \mathbb{R}$. Such functions are the Hamiltonian lifts of left-invariant vector fields on $S E(3)$, because $p\left(g(t) A_{i}\right)=\hat{p}\left(A_{i}\right)$ for any $p=(g(t), \hat{p})$ and any $A_{i} \in \mathfrak{s e}(3)$. Moreover, if $M_{i}, p_{i}$ is a collection of linear functions generated by the basis $A_{i}, B_{i}$ in $\mathfrak{s e}(3)$ then the vector $\left(M_{1}, M_{2}, M_{3}, p_{1}, p_{2}, p_{3}\right)$ is the coordinate vector of $\hat{p}$ relative to the dual basis $A_{i}^{*}, B_{i}^{*}$. Therefore, the Hamiltonian (11) can be expressed on $\mathfrak{s e}^{*}(3)$ as

$$
H=p_{1}+k_{1} M_{1}+k_{2} M_{2}-\frac{1}{2}\left(k_{1}^{2}+k_{2}^{2}\right)
$$

Then through the Maximum principle of optimal control and the fact that the control Hamiltonian (12) is a concave function of the control functions $k_{1}, k_{2}$, it follows from [12] that calculating $\frac{\partial H}{\partial k_{1}}=\frac{\partial H}{\partial k_{2}}=0$ yields the optimal controls:

$$
k_{1}=M_{1}, k_{2}=M_{2}
$$

substituting (13) into (12) gives the optimal Hamiltonian:

$$
H=p_{1}+\frac{1}{2}\left(M_{1}^{2}+M_{2}^{2}\right)
$$

In addition substituting the expressions (13) into (4) the optimal (with respect to the cost function (8)) motions are the solutions $g(t) \in S E(3)$ of the differential equation:

$$
\frac{d g(t)}{d t}=g(t)\left(\begin{array}{cccc}
0 & 0 & 0 & 0 \\
1 & 0 & -M_{1} & -M_{2} \\
0 & M_{1} & 0 & 0 \\
0 & M_{2} & 0 & 0
\end{array}\right)
$$

Therefore, the path planning problem amounts to solving the extremal curves $M_{1}$ and $M_{2}$ and then integrating equation (15) to obtain the optimal motions $g(t) \in S E(3)$. In Section IV we solve the extremal curves $M_{1}$ and $M_{2}$ explicitly for a special case and then proceed in Section $\mathrm{V}$ to solve the equation (15) for $g(t) \in S E(3)$.

\section{SOlving THE EXTREMAL CURVES}

In order to solve equation (15) it is necessary to solve the extremals curves $M_{1}$ and $M_{2}$. In order to do this we need to look at all the available information. Firstly, we compute the corresponding Hamiltonian vector fields from the leftinvariant Hamiltonian (14). To do this it is convenient to state the Lie bracket table with the Lie bracket defined by $[X, Y]=X Y-Y X$, then for the basis (6):

\begin{tabular}{|c|c|c|c|c|c|c|}
\hline$[]$, & $A_{1}$ & $A_{2}$ & $A_{3}$ & $B_{1}$ & $B_{2}$ & $B_{3}$ \\
\hline$A_{1}$ & 0 & $A_{3}$ & $-A_{2}$ & 0 & $-B_{3}$ & $B_{2}$ \\
$A_{2}$ & $-A_{3}$ & 0 & $A_{1}$ & $B_{3}$ & 0 & $-B_{1}$ \\
$A_{3}$ & $A_{2}$ & $-A_{1}$ & 0 & $-B_{2}$ & $B_{1}$ & 0 \\
$B_{1}$ & 0 & $-B_{3}$ & $B_{2}$ & 0 & 0 & 0 \\
$B_{2}$ & $B_{3}$ & 0 & $-B_{1}$ & 0 & 0 & 0 \\
$B_{3}$ & $-B_{2}$ & $B_{1}$ & 0 & 0 & 0 & 0 \\
\hline
\end{tabular}

the Poisson bracket is given by the equation:

$$
\{\hat{p}(\cdot), \hat{p}(\cdot)\}=-\hat{p}([\cdot, \cdot])
$$

and it follows that the time derivative of $M_{1}$ along the Hamiltonian flow is then:

$$
\begin{aligned}
& \dot{M}_{1}=\left\{M_{1}, H\right\} \\
& =\left\{M_{1}, p_{1}\right\}+M_{1}\left\{M_{1}, M_{1}\right\}+M_{2}\left\{M_{1}, M_{2}\right\} \\
& =0+0-M_{2} M_{3}
\end{aligned}
$$

the remaining Hamiltonian vector fields are:

$$
\begin{aligned}
& \dot{M}_{1}=-M_{2} M_{3} \\
& \dot{M}_{2}=-p_{3}+M_{1} M_{3} \\
& \dot{M}_{3}=p_{2} \\
& \dot{p}_{1}=M_{2} p_{3} \\
& \dot{p}_{2}=p_{1} M_{3} \\
& \dot{p}_{3}=-p_{1} M_{1}
\end{aligned}
$$


The integral curves of the Hamiltonian vector fields (18) are necessary conditions for optimality and can be used to solve for our critical variables $M_{1}$ and $M_{2}$. In addition to this there are constants of motions inherent in all left-invariant Hamiltonian systems defined on $S E(3)$, see [12] for details. In particular the Casimir functions

$$
I_{2}=p_{1}^{2}+p_{2}^{2}+p_{3}^{2}
$$

and

$$
I_{3}=M_{1} p_{1}+M_{2} p_{2}+M_{3} p_{3}
$$

are constant along the Hamiltonian flow. This is easily verified as they are in involution with any other constant function $C_{i}$ on $\mathfrak{s e}^{*}(3)$ i.e. $\left\{I_{2}, H\right\}=0,\left\{I_{3}, H\right\}=0$ and $\left\{I_{2}, I_{3}\right\}=0$, see [12] for details. Using the Hamiltonian vector fields and the constants of motion $H, I_{2}, I_{3}$ we can attempt to solve for $M_{1}$ and $M_{2}$. Solving the Hamiltonian vector fields (18) with respect to the constants of motion can be achieved numerically using such methods as those described in [22]. However, the advantage of studying Hamiltonian systems is that in many cases analytic solutions can be computed, even for high dimensional cases such as our system defined on the 12 -D cotangent bundle $T^{*} S E(3) \cong S E(3) \times \mathfrak{s e}^{*}(3)$. Moreover, Hamiltonian systems that can be solved analytically are called Integrable Hamiltonian systems, explicitly from [23]: A Hamiltonian function on a symplectic manifold $N$ of dimension $2 n$ is said to be integrable if there exist constant functions $\varphi_{2}, \ldots, \varphi_{n}$ on $N$ that together with the Hamiltonian $H=\varphi_{1}$ satisfy the following two properties:

- $\varphi_{1}, \ldots, \varphi_{n}$ are functionally independent i.e the differentials $d \varphi_{1}, \ldots, d \varphi_{n}$ are linearly independent for an open subset of $N$.

- The functions $\varphi_{1}, \ldots, \varphi_{n}$ Poisson commute with each other.

Thus, in identifying the $(n-1)$ functions $\varphi_{i}$ the Hamiltonian function is completely integrable and analytic solutions of the Hamiltonian vector fields can be found. For left-invariant Hamiltonian systems defined on $S E(3)$ we can be more specific about integrability, following the arguments posed in [23] we state the following theorem:

Theorem 1: For any left (respectively right) invariant Hamiltonian system defined on $S E(3)$, there exist five constants of motion $\varphi_{1}=H, \varphi_{2}=I_{2}, \varphi_{3}=I_{3}, \varphi_{4}, \varphi_{5}$, where the constants of motion $\varphi_{4}, \varphi_{5}$ correspond to right-invariant vector fields.

Proof. see [23]. It follows from Theorem 1 that for the left-invariant Hamiltonian (14) and the corresponding vector fields (18) to be integrable, an additional constant of motion needs to be identified. This is because the Hamiltonian is defined on the 12-D cotangent bundle $T^{*} S E(3) \cong S E(3) \times$ $\mathfrak{s e}^{*}(3)$ and therefore six constants of motion are required for integrability. In the classic example of Hamiltonian systems defined on $S E(3)$ such as the spinning top [12], the additional constant of motion required for integrability arises through the assumption that the top is axially symmetric. However, an analogous symmetry argument can not be used for the Hamiltonian vector fields (18). Therefore, to solve (18) numerical methods such as those described in [22] can be used to solve the extremal curves. However, using numerical methods does not provide a global solution to this system as these methods are inherently local. Computing analytic solutions are highly desirable in motion planning as they lend themselves to conceptually clear global investigations. In the remainder of this paper a trivial integrable case will be stated followed by a non-trivial integrable case of the Hamiltonian vector fields (18). For the non-trivial case analytic expressions are derived for the optimal motions.

A trivial example of an integrable case of vector fields (18) occurs when $p_{1}=p_{2}=p_{3}=M_{1}=M_{2}=M_{3}=0$. Moreover, for these values $p_{1}=p_{2}=p_{3}=M_{1}=M_{2}=M_{3}$ are constant $\forall t$ and therefore the system is integrable (providing 6 constants necessary for integrability). Substituting these values into (9) yields

$$
\frac{d g(t)}{d t}=g(t) B_{3}
$$

this is easily integrated to yield $\gamma(t)=[t, 0,0]^{T}$ with $R$ equal to a $3 \times 3$ matrix with zero entries. Therefore, a straight line motion with zero rotation about this line is an optimal rigid body motion. In addition there exists a non-trivial integrable case of the Hamiltonian vector fields (18). This case is considered non-trivial as it gives rise to time-dependent extremal curves. It is observed that $p_{1}=p_{2}=p_{3}=0$ is an invariant surface for the Hamiltonian vector fields (18). Explicitly, for $p_{1}=p_{2}=p_{3}=0$ the equations (18) degenerate to:

$$
\begin{aligned}
& \dot{M}_{1}=-M_{2} M_{3} \\
& \dot{M}_{2}=M_{1} M_{3} \\
& \dot{M}_{3}=0 \\
& \dot{p}_{1}=0 \\
& \dot{p}_{2}=0 \\
& \dot{p}_{3}=0
\end{aligned}
$$

this implies that $M_{3}$ (if not identically zero), which is functionally independent of $H, I_{2}, I_{3}$ is constant. For convenience the constant $M_{3}$ will be denoted by c. In addition $p_{1}=p_{2}=$ $p_{3}=0 \forall t$. It follows that the Hamiltonian vector fields (22) are completely integrable. These particular curves will be the focus of the remainder of this paper. For these particular curves the Hamiltonian (14) reduces to

$$
H=M_{1}^{2}+M_{2}^{2}
$$

and we assume that $H>0$. It follows parameterizing the Hamiltonian (23) using polar coordinates in such a way that the differential equations (22) are satisfied that the extremal curves are:

$$
\begin{aligned}
& M_{1}=r \cos c t \\
& M_{2}=r \sin c t \\
& M_{3}=c
\end{aligned}
$$

It follows that as $\mathfrak{s e}^{*}(3) \cong \mathbb{R}^{6}$, the extremal curves can be geometrically interpreted as a circle embedded in $\mathbb{R}^{6}$. Substituting $M_{1}=M_{2}=0$ into (15), it is easily shown that the corresponding optimal motions are along a straight line 
with zero rotation about this line. However, to compute the optimal motions corresponding to the extremal curves (24) is not trivial as the elements of the Lie algebra are timedependent. Integrating (15), where $M_{1}$ and $M_{2}$ are defined by (24) is the subject of the next section.

\section{OPTIMAL MOTIONS FOR THE RIGID BODY}

Having solved for the extremal curves $M_{1}$ and $M_{2}$ we now wish to solve for the corresponding optimal motions in $S E(3)$. This is done by integrating the equation (15), which describes optimal motions with respect to the cost function (8). For the purpose of integration it is convenient to split the natural frame (15) into its translational and rotational part:

$$
\frac{d \gamma(t)}{d t}=R \vec{e}_{1}
$$

and

$$
\frac{d R}{d t}=R\left(\begin{array}{ccc}
0 & -M_{1} & -M_{2} \\
M_{1} & 0 & 0 \\
M_{2} & 0 & 0
\end{array}\right)
$$

where $R^{-1}=R^{T}$. This section exploits the conservation laws inherent in all left-invariant Hamiltonian systems on $S E(3)$ to integrate (25) and (26). For convenience we define a basis for the Lie algebra $\mathfrak{s o}(3)$

$$
\begin{gathered}
E_{1}=\left(\begin{array}{ccc}
0 & -1 & 0 \\
1 & 0 & 0 \\
0 & 0 & 0
\end{array}\right), E_{2}=\left(\begin{array}{ccc}
0 & 0 & -1 \\
0 & 0 & 0 \\
1 & 0 & 0
\end{array}\right) \\
E_{3}=\left(\begin{array}{ccc}
0 & 0 & 0 \\
0 & 0 & -1 \\
0 & 1 & 0
\end{array}\right)
\end{gathered}
$$

then the following quantities are conserved for all leftinvariant Hamiltonian systems on $S E(3)$

$$
R P R^{-1}=\text { constant }
$$

and

$$
R M R^{-1}+\left[X, R P R^{-1}\right]=\text { constant }
$$

where

$$
\begin{aligned}
& M=M_{1} E_{1}+M_{2} E_{2}+M_{3} E_{3} \\
& P=p_{1} E_{1}+p_{2} E_{2}+p_{3} E_{3} \\
& X=x_{1} E_{1}+x_{2} E_{2}+x_{3} E_{3}
\end{aligned}
$$

where $x_{1}, x_{2}, x_{3}$ are the position coordinates of the vector $\gamma(t)=\left[x_{1}, x_{2}, x_{3}\right]^{T}$. For a proof that the quantities (28) and (29) are constant for all left-invariant Hamiltonian systems on $S E(3)$ see [12]. Using these constants of motion we are able to integrate (26) which is stated in the following theorem:

Theorem 2: $R=(x|y| z) \in S O(3)$ is the rotation matrix which relates the natural frame to a fixed inertial frame where:

$$
\begin{aligned}
& x=\left[\begin{array}{c}
\frac{c}{K} \\
-\frac{r}{K} \sin K t \\
\frac{r}{K} \cos K t
\end{array}\right] \\
& y=\left[\begin{array}{c}
\frac{r}{K} \sin c t \\
\cos K t \cos c t+\frac{c}{K} \sin K t \sin c t \\
\sin K t \cos c t-\frac{c}{K} \cos K t \sin c t
\end{array}\right] \\
& z=\left[\begin{array}{c}
-\frac{r}{K} \cos c t \\
-\frac{c}{K} \cos c t \sin K t+\sin c t \cos K t \\
\frac{c}{K} \cos K t \cos c t+\sin c t \sin K t
\end{array}\right]
\end{aligned}
$$

where $K=\sqrt{r^{2}+c^{2}}$ and $r, c$ are the constant parameters of the curvatures(24)

Proof.

For these particular curves $p_{1}=p_{2}=p_{3}=0$ the conservation laws (28) and (29) reduce to:

$$
R M R^{-1}=\text { constant }
$$

this constant matrix $R M R^{-1}$ is then conjugated for a particular solution $R$ such that:

$$
R M R^{-1}=\sqrt{M_{1}^{2}+M_{2}^{2}+M_{3}^{2}} E_{3}
$$

substituting (24) into (33) gives:

$$
R M R^{-1}=\sqrt{r^{2}+c^{2}} E_{3}
$$

for convenience we will define the constant $K^{2}=r^{2}+c^{2}$ therefore we can write

$$
M=K R^{-1} E_{3} R
$$

expressing $R$ in a convenient coordinate form [12]:

$$
R=\exp \left(\phi_{1} E_{3}\right) \exp \left(\phi_{2} E_{2}\right) \exp \left(\phi_{3} E_{3}\right)
$$

and substituting (36) into (35) yields:

$$
M=K \exp \left(-\phi_{3} E_{3}\right) \exp \left(-\phi_{2} E_{2}\right) E_{3} \exp \left(\phi_{2} E_{2}\right) \exp \left(\phi_{3} E_{3}\right)
$$

explicitly

$$
M=K\left(\begin{array}{ccc}
0 & \cos \phi_{3} \sin \phi_{2} & -\sin \phi_{2} \sin \phi_{3} \\
-\cos \phi_{3} \sin \phi_{2} & 0 & -\cos \phi_{2} \\
\sin \phi_{2} \sin \phi_{3} & \cos \phi_{2} & 0
\end{array}\right)
$$

equating $M$ in (30) to (38) gives:

$$
\begin{aligned}
& M_{1}=-K \cos \phi_{3} \sin \phi_{2} \\
& M_{2}=K \sin \phi_{2} \sin \phi_{3} \\
& M_{3}=K \cos \phi_{2}
\end{aligned}
$$

therefore it is easily shown that:

$$
\begin{aligned}
& \cos \phi_{2}=\frac{M_{3}}{K} \\
& \sin \phi_{2}= \pm \sqrt{1-\frac{M_{3}^{2}}{K^{2}}}
\end{aligned}
$$

and substituting (24) into (40) and simplifying gives:

$$
\begin{aligned}
\cos \phi_{2} & =\frac{c}{K} \\
\sin \phi_{2} & = \pm \frac{r}{K}
\end{aligned}
$$


in addition from (39) we have:

$$
\tan \phi_{3}=-\frac{M_{2}}{M_{1}}
$$

therefore:

$$
\begin{aligned}
& \sin \phi_{3}=\mp \frac{M_{2}}{\sqrt{M_{1}^{2}+M_{2}^{2}}} \\
& \cos \phi_{3}= \pm \frac{M_{1}}{\sqrt{M_{1}^{2}+M_{2}^{2}}}
\end{aligned}
$$

and substituting (24) into (43) and simplifying yields:

$$
\begin{aligned}
& \sin \phi_{3}=\mp \sin c t \\
& \cos \phi_{3}= \pm \cos c t
\end{aligned}
$$

in order to obtain an expression for $\phi_{1}$ we substitute (36) into (26) and simplify to yield:

$$
\begin{aligned}
& \dot{\phi}_{1}\left(\begin{array}{ccc}
0 & \cos \phi_{3} \sin \phi_{2} & -\sin \phi_{2} \sin \phi_{3} \\
-\cos \phi_{3} \sin \phi_{2} & 0 & -\cos \phi_{2} \\
\sin \phi_{2} \sin \phi_{3} & \cos \phi_{2} & 0
\end{array}\right) \\
&+ \dot{\phi}_{2}\left(\begin{array}{ccc}
0 & -\sin \phi_{3} & -\cos \phi_{3} \\
\sin \phi_{3} & 0 & 0 \\
\cos \phi_{3} & 0 & 0
\end{array}\right) \\
&+\dot{\phi}_{3}\left(\begin{array}{ccc}
0 & 0 & 0 \\
0 & 0 & -1 \\
0 & 1 & 0
\end{array}\right)=\left(\begin{array}{ccc}
0 & -M_{1} & -M_{2} \\
M_{1} & 0 & 0 \\
M_{2} & 0 & 0
\end{array}\right)
\end{aligned}
$$

which leads to

$$
\dot{\phi}_{1} \sin \phi_{2} \sin \phi_{3}+\dot{\phi}_{2} \cos \phi_{3}=M_{2}
$$

and

$$
\dot{\phi}_{1} \cos \phi_{3} \sin \phi_{2}-\dot{\phi}_{2} \sin \phi_{3}=-M_{1}
$$

dividing equation (47) by $\sin \phi_{3}$ and (46) by $\cos \phi_{3}$ and adding the two equations and simplifying yields:

$$
\dot{\phi}_{1}=\frac{M_{2} \sin \phi_{3}-M_{1} \cos \phi_{3}}{\sin \phi_{2}}
$$

substituting (24), (41) and (44) into (48) and integrating with respect to $t$ yields:

$$
\phi_{1}=K t+\beta
$$

where $\beta$ is a constant of integration. Assuming for simplicity of exposition that $\beta=0$ and substituting (41), (44), (49) into (36) and choosing the natural frame to be positively oriented yields (31) $R=(x|y| z)$

Having solved for $\phi_{1}, \phi_{2}, \phi_{3}$, we can easily solve (25) for $\gamma(t) \in \mathbb{R}^{3}$, which is stated as a Lemma:

Lemma 1: The optimal path $\gamma(t) \in \mathbb{R}^{3}$ defined by the differential equation (25), with $M_{1}=r \cos c t$ and $M_{2}=r \sin c t$ are helices described by:

Proof.

$$
\gamma(t)=\frac{1}{K^{2}}[c t, r \cos K t, r \sin K t]^{T}
$$

Substituting (36) into (25) yields:

$$
\frac{d \gamma(t)}{d t}=\left(\begin{array}{c}
\cos \phi_{2} \\
-\sin \phi_{1} \sin \phi_{2} \\
\cos \phi_{1} \sin \phi_{2}
\end{array}\right)
$$

then substituting (49) and (41) into (51) and assuming a positively oriented frame gives:

$$
\frac{d \gamma(t)}{d t}=\frac{1}{K}\left(\begin{array}{c}
c \\
-r \sin K t \\
r \cos K t
\end{array}\right)
$$

finally on integrating (52) yields

$$
\gamma(t)=\frac{1}{K} \int\left(\begin{array}{c}
c \\
-r \sin K t \\
r \cos K t
\end{array}\right) d t=\frac{1}{K^{2}}[c t, r \cos K t, r \sin K t]^{T}
$$

Therefore, this particular smooth rigid body motion traces a helix and the natural frame rotates along with this helical motion according to the rotation matrix $R=(x|y| z) \in S O(3)$ in (31). These motions are also expressed completely in terms of the parameters $r, c$ of the geometrically invariant natural curvatures $k_{1}=r \cos c t$ and $k_{2}=r \sin c t$ and therefore independently of a coordinate frame. It is important to note that although screw motions trace helical paths, we cannot say that the particular solution (50) is a screw motion. A general screw motion can be described by motion along a straight line with rotation about the direction of motion. However, because in general, framed curves do not coincide with the rigid body fixed frame we cannot conclude that the rigid body is rotating in a screw motion. Indeed, in the particular case that the rigid body is constrained to travel in the direction of the unit tangent vector to the curve, we can say that the solution (50) is not a screw motion. In this case the motion is along a helix (not a straight line) with rotation about this helix.

Note that the optimal motion described by (50) and (31) is only a particular optimal motion with initial $\gamma(0)$ and $R_{0}=R(0)$ chosen to satisfy the equation (33). Moreover, expressing these initial conditions as an element of $S E(3)$ at $t=0$ the initial $g(0) \in S E(3)$ is equal to:

$$
g(0)=\left(\begin{array}{cccc}
1 & 0 & 0 & 0 \\
0 & \frac{c}{K} & 0 & -\frac{r}{K} \\
\frac{r}{K^{2}} & 0 & 1 & 0 \\
0 & \frac{r}{K} & 0 & \frac{c}{K}
\end{array}\right)
$$

however, from the particular optimal motion (50) and (31) it is easy to express a more general optimal motion given any initial condition. Firstly, define arbitrary initial position and orientation by $g_{\text {int }} \in S E(3)$ and the position and orientation at time $t$ given these initial conditions as $g_{\text {gen }} \in S E(3)$. It follows that given a particular solution $g(t) \in S E(3)$ where $g(0) \in S E(3)$ is $g(t) \in S E(3)$ at $t=0$, the general solution $g_{\text {gen }} \in S E(3)$ can be expressed as:

$$
g_{\text {gen }}=g_{\text {int }} g(0)^{-1} g(t)
$$

$g(0)^{-1}$ is the inverse of $g(0)$ such that $g(0)^{-1} g(0)=I$ where $I$ is the identity matrix. Computing the general solution explicitly from the particular optimal motion described by (50) and (31) yields:

$$
g_{\text {gen }}=g_{\text {int }}\left(\begin{array}{cc}
1 & 0 \\
\gamma(t) & R(t)
\end{array}\right)
$$


where

$$
\gamma(t)=\left[\begin{array}{c}
\frac{c^{2} t+r^{2} \sin (K t)}{K\left(c^{2}+r^{2}\right)} \\
\frac{r(\cos (K t)-1)}{c^{2}+r^{2}} \\
\frac{c r(\sin (K t)-t)}{K\left(c^{2}+r^{2}\right)}
\end{array}\right]
$$

and $R(t)=(x|y| z)$ is:

$$
\begin{aligned}
& x=\left[\begin{array}{c}
\frac{c^{2}+r^{2} \cos (K t)}{c^{2}+r^{2}} \\
\frac{-(r+K r \sin (K t))}{c^{2}+r^{2}} \\
\frac{-2 c r \sin ^{2}(K t / 2)}{c^{2}+r^{2}}
\end{array}\right] \\
& y=\left[\begin{array}{c}
\frac{r((c-c \cos (K t)) \sin (c t)+K \cos (c t) \sin (K t))}{c^{2}+r^{2}} \\
\frac{K^{2} \cos (c t) \cos (K t)+c K \sin (c t) \sin (K t)-r}{c^{2}+r^{2}} \\
\frac{\left.-\left(r^{2}+c^{2} \cos (K t)\right) \sin (c t)+c K \cos (c t) \sin (K t)\right)}{c^{2}+r^{2}}
\end{array}\right] \\
& z=\left[\begin{array}{l}
\frac{r(c \cos (c t)(\cos (K t)-1)+K \sin (c t) \sin (K t))}{c^{2}+r^{2}} \\
\frac{-\left(r+c K \cos (c t) \sin (K t)-K^{2} \sin (c t) \sin (K t)\right)}{c^{2}+r^{2}} \\
\frac{\left.\cos (c t)\left(r^{2}+c^{2} \cos (K t)\right)+c K \sin (c t) \sin (K t)\right)}{c^{2}+r^{2}}
\end{array}\right]
\end{aligned}
$$

this optimal helical motion describes a curve through any specified initial position and orientation of a rigid body and can be used to plan motions adjusting the parameters $r$ and $c$ as required.

\section{CONCLUSION}

This paper derives smooth, optimal trajectories on the Euclidean group of motions $S E(3)$. These trajectories have the advantage that they are expressed independently of a local coordinate frame. In order to compute such trajectories we assign a geometric frame to the rigid body, that is, a moving frame along a space curve. A point in the rigid body follows a curve and an orthonormal frame attached to the rigid body stays aligned with this geometric frame. We use the Natural Frame, where the parameters of the motion are given by the natural curvatures of the space curve along which the object moves. The problem of computing smooth trajectories was then formulated as an optimal control problem where the cost function to be minimized is the integral of the curvature squared. This problem is analogous to the elastic problem from differential geometry and therefore the resulting rigid body motions will trace elastic curves. An application of the Maximum Principle to this optimal control problem results in a system of first order differential equations that yield coordinate free necessary conditions for optimality. We analysed a particular set of curves that satisfy these necessary conditions and provide analytic solutions for the corresponding optimal motions. Moreover, this paper derives helical motions that are expressed completely in terms of their natural curvatures and as such provide smooth, optimal curves described in a coordinate free manner that can be used to plan rigid body motions.

\section{REFERENCES}

[1] Leonard, N., Krishnaprasad, P. S., 'Motion control of drift free, leftinvariant systems on Lie groups'. IEEE Transactions on Automatic control, 1995.
[2] Justh, E. W., Krishnaprasad, P. S., 'Natural frames and interacting particles in three dimension'. Proceedings of 44th IEEE Conference on Decision and Control and the European Control Conference, 2005.

[3] Duric, Z., Rivlin, E., Rosenfeld, A., 'Understanding object motion'. Image and Vision Computing 16, pp. 785-797, 1998.

[4] Latcombe, J. C., 'Robot Motion Planning'. Boston, Kluwer Academic Publishers, 1991

[5] Whitney, D. E., 'The mathematics of coordinated control of prosthestic arms and manipulators'. ASME Journal of Dynamical Systems and Control, vol. 94, 1972.

[6] Waldron, K. J., 'Geometrically based manipulator rate control algoritms'. Mechanism and machine theory, vol. 17, no. 6, 1982.

[7] Crouch, P., Silva Leite, F., 'The Dynamic interpolation problem: On Riemannian manifolds, Lie groups and Symmetric spaces'. Journal of Dynamical and control systems, Vol.1, No. 2, p177-202, 1995.

[8] Noakes, L., Heinzinger, G., and Paden, B., 'Cubic Splines on curved Spaces'. IMA Journal of Mathematical Control and Information 1989 6(4):465-473

[9] Zefran, M., Kumar, V., Croke, C., 'Choice of Riemannian metrics for rigid body kinematics'. ASME 24th Biennial Mechanisms Conference, 1996.

[10] Zefran, M., Kumar, V., Croke, C., 'On the generation of smooth three dimensional rigid body motions'. IEEE Trans. Robotics and Automation, vol. 14, pp. 579-589, 1995.

[11] Jurdjevic, V., Monroy-Perez, F., 'Variational Problems on Lie Groups and their Homogeneous Spaces: Elastic Curves, Tops, and constrained Geodesic Problems in Nonlinear Geometric Control Theory'. World Scientific, 2002.

[12] Jurdjevic, V., 'Geometric Control Theory'. Advanced Studies in Mathematics, Cambridge University Press, 52,1997.

[13] Sussmann, H. J., 'An introduction to the coordinate-free maximum principle'. In Geometry of Feedback and Optimal Control,B. Jakubczyk and W. Respondek Eds., Marcel Dekker, New York, pp. 463-557, 1997.

[14] Bottema, O., Roth, B.,'Theoretical Kinematics'. Dover Publications, New York, 1990.

[15] Wagner, M. G., Ravani, B., 'Curves with rational Frenet-Serret motion’. Computer Aided Geometric Design, vol. 15, pp. 79-101, 1997.

[16] Bishop, R.L., 'There is more than one way to frame a curve'. Am. Math. Monthly, vol. 82, pp.246-251, 1975.

[17] Ravani, R., Meghadari, A., 'Spatial rational motions based on FrenetSerret Curves'. Proceedings of IEEE Conference on Intl. Conf. Systems, Man and Cybernetics, pp. 4456-4461, 2004.

[18] Ravani, R., Meghadari, A., 'Spatial rational motions based on FrenetSerret Curves'. Proceedings of IEEE Conference on Intl. Conf. Systems, Man and Cybernetics, pp. 4456-4461, 2004.

[19] Krause, J., 'Some Remarks on Orthonormal Tetrad Transport'. International Journal of Theoretical Physics, Vol. 12, No. 1, pp. 35-45, 1975.

[20] Bliss, A., 'Lectures on the Calculus of Variations'. Univ. Chicago Press, 1946.

[21] Bryant, R., and Hsu, L. 'Rigidity of integral curves of rank 2 distribution'. Duke Univ. Preprint, No.5, 1993.

[22] Hairer, E., Lubich, C., Wanner, G., 'Geometric Numerical Integration: Structure preserving algorithms for Ordinary Differential Equations'. Springer, 2002.

[23] Jurdjevic, V., 'Integrable Hamiltonian Systems on Complex Lie groups'. in Memoirs of the American Mathematical Society, Providence, RI: The American Mathematical Society, Vol. 178, 2005.

[24] Bloch, A. M., 'Nonholonomic Mechanics and Control'. SpringerVerlag, New York,2003. 